\title{
A UHF/UWB hybrid silicon RFID tag with on-chip antennas
}

\author{
Philipp K Gentner ${ }^{1 *}$, Robert Langwieser ${ }^{1}$, Arpad L Scholtz ${ }^{1}$, Günter Hofer ${ }^{2}$ and Christoph F Mecklenbräuker ${ }^{1}$
}

\begin{abstract}
In this contribution, we describe and analyse a miniature wireless radio frequency identification (RFID) chip with on-chip antennas (OCA) and ultra wideband (UWB) signalling by real-world measurements. With the on-chip antenna approach, no external antennas are required, and the size of the overall tag is identical to the size of the chip alone ( $3.5 \mathrm{~mm} \times 1 \mathrm{~mm}$ ). The chip is powered through inductive coupling and controlled by an RFID signal at $866 \mathrm{MHz}$ in the downlink, while the uplink transmits a quaternary pulse-position-modulated (4-PPM) UWB signal at $5.64 \mathrm{GHz}$ with pulses having a duration in the order of nanoseconds. In this contribution, the hybrid or asymmetric communication scheme between prototype chip and reader, the embedded OCA, and the measurement setup are described. The prototype achieves 4-PPM bit rate of $126 \mathrm{Mbit} / \mathrm{s}$ based on a pulse-train transmission with a duration of $10 \mu \mathrm{s}$. The small size, high data rate, and fine time resolution of the UWB impulse radio offer new features and sensing capabilities for future RFID-like applications.
\end{abstract}

\section{Introduction}

Tiny systems on chip (SoC) with on-chip antennas (OCAs) are useful if a transmission of data is necessary over a short distance. Especially for radio frequency identification (RFID) systems, the limited communication range increases security and protects privacy.

Small tags combined with sensing capabilities, such as temperature or shock sensors, allow the tracking of individual items for example frozen goods in a cold chain. Due to their small size, the systems on chip can be placed on or in objects while reducing the cost by avoiding bulky antennas.

In this contribution, a fully integrated system on chip is presented, which features on-chip antennas for a hybrid or in other words asymmetric communication scheme. It gives insight into our past achievements and current studies in the field of short range connectivity devices for RFID applications or for wireless sensors. At first, the scheme is explained in detail in section "Asymmetric communication scheme" with a survey of impulse transmitters and related achievements in research. Then our manufactured ultra-high frequency/ultra-wideband (UHF/UWB) hybrid

\footnotetext{
${ }^{*}$ Correspondence: philipp.gentner@nt.tuwien.ac.at

${ }^{1}$ Institute of Telecommunications, Vienna University of Technology,

Gusshausstrasse 25/E389, Vienna 1040, Austria

Full list of author information is available at the end of the article
}

system on chip is introduced, with all its components and a focus on the on-chip antennas used, a dual antenna concept with an electric and a magnetic antenna.

After the description of the device under test, the development of the measurement setup is explained in detail, and also, results are presented in the time and the frequency domains. The decoding of the received wideband data signal from the hybrid UHF/UWB tag is shown. Before the conclusion, the transmission loss between the impulse-transmitting OCA and the according uplink reader antenna is calculated from measurements.

\section{Asymmetric communication scheme}

The most famous example of an asymmetric communication scheme is used everyday all over the world through browsing of the web. In the downlink from the provider to the user, a much higher amount of data is needed by pictures, movies, or text. In the uplink, mostly control or request data are sent from a personal computer to the provider. This idea is also used in long-term evolution (LTE), where the downlink peak rates are higher as those in the uplink.

In the case of RFID or sensor applications, the high data rate is shifted to the uplink. Since the tags or sensors are very small and the available power has to be used as efficiently as possible, it is advantageous to have a fast

\section{是 Springer}

(c) 2013 Gentner et al: licensee Springer. This is an Open Access article distributed under the terms of the Creative Commons Attribution License (http://creativecommons.org/licenses/by/2.0), which permits unrestricted use, distribution, and reproduction in any medium, provided the original work is properly cited. 
transmission in the uplink. The downlink, from a reader station to a sensor or tag, provides the energy, control commands, and clock for synchronisation in a UHF band, with the data rate being usually lower. A concept of an asymmetric communication scheme for RFID scenarios was introduced by Zheng et al. in [1] and has been manufactured and characterised in the works of Baghaei-Nejad and Radiom et al. in [2] and [3].

In the papers mentioned above, impulse radio UWB is considered as the appropriate scheme for the uplink, being able to provide a fast data rate and also being more power efficient. This has been shown by Calhoun et. al in [4], who summarise the necessary energy per pulse for UWB transmitters of recent research. The authors found that the energy per pulse is lower than $10 \mathrm{~nJ} / \mathrm{bit}$ and constant over a data rate from $1 \mathrm{Mbit} / \mathrm{s}$ to $10 \mathrm{Gbit} / \mathrm{s}$, and compares it with the energy per bit for a bluetooth transmitter being typically $25 \mathrm{~nJ} /$ bit.

In our opinion, transmitting pulses in the baseband is not a good choice and should not be considered for the application in mind. The reasons are the following:

- The powering UHF field interferes with the spectrum of pulses transmitted in the baseband, which leads to an increased reader complexity.

- Reading multiple tags at the same time leads to collisions. Using modulated pulses, the probability of collisions can be reduced by changing the modulation frequency.

- A small size reader antenna is important for many applications.

- Using on-chip antennas with their small aperture, uplink frequencies in the C- or X-band are preferred.

Non-baseband impulse radio UWB transmitters can be realised in two ways [5]: firstly, baseband pulses can be upconverted with the aid of a local oscillator. Secondly, pulse creation, e.g. with delay lines following pulse shaping filter can be employed. In terms of reduced transmitter power consumption, a pulse-position modulation scheme is very promising because it allows to transmit multiple bits per pulse.

Impulse radio as an additional asset of a tag introduces a multitude of new possibilities and applications for RFID scenarios [6]. In recent UWB research, indoor localisation is studied extensively for example by Meissner et al. in [7]. Concerning localisation, the IEEE standard 802.15.4a provides a good basis for commercial applications. Zheng et al. describe in [8] a transceiver achieving a ranging accuracy of $3 \mathrm{~cm}$. Readers for localisation will very likely use an array antenna to track the small device. In [9] and [10], UWB antenna arrays are introduced and analysed concerning their ability to operate in a wideband spectrum and are consecutively used to operate with the wideband tags.
Two inductively coupled magnetic loop antennas offer a wireless power transfer technique, which is used successfully for medical implants [11], such as pacemakers and biosensors, or for charging electric vehicles [12]. An RFID tag with an asymmetric communication scheme favourably should store energy from an energy source to be able to transmit pulses. Here, power-scavenging units harvest energy from the narrowband UHF downlink signal. Figures of merits are the efficiency of the rectifier and the value of the capacitor, where the harvested energy is stored. The downlink antenna will usually be an inductive antenna with high Q-factor, whereas a wideband antenna with low $\mathrm{Q}$-factor is required in the uplink.

\section{Hybrid UHF/UWB system on chip}

In our approach towards a short-range connectivity device, we started with externally powered complementary metal oxide semiconductor substrate (CMOS) structures (see Figure 1). These CMOS prototypes with OCAs were controlled and powered through bondwires. In order to compare the radiation of different types of OCAs, a voltage-controlled oscillator ( $\mathrm{VCO}$ ) was swept over a huge frequency range of several octaves [13]. Furthermore, nanosecond pulses were transmitted with a simple on-off keying pulse transmitter - created by a glitch generator, whose output was multiplied with the VCO frequency. A proof of concept concerning the energy transfer was done, with inductive coupling using a magnetic OCA [14]. With a modulated backscatter technique, the voltage induced into the miniaturised loop antenna was analysed over area and over distance. The power harvesting concept was studied and characterised with a small $3 \mathrm{~mm} \times 1 \mathrm{~mm}$ system, where two different OCAs were used for receiving energy and for transmitting data [15]. The powerharvesting concept must be able to collect power of some $\mu \mathrm{W}$ in the long term. This energy, stored in an efficient way with on-chip batteries or capacitors, is used for driving the UWB front end, which requires some $\mathrm{mW}$ within a short period of time. The asymmetric scheme allows to replace the used harvesting concept, if clock and data for configuring the chip is provided through another communication channel. Also, this scheme allows to investigate the performance of on-chip antennas without having a galvanic connection. In the course of these investigations, we observed that bondwires used in earlier work have a huge influence due to coupling. Bondwires, typically the same size and in close vicinity of the chip, act as a high Q-factor antenna, and support either the transmitted pulses or the energy-harvesting path. In the downlink path, a coupling into the chips' $V_{\mathrm{SS}}$ is established, which yields to a better efficiency of the rectifier. Using plane OCAs, the energy received by the reader (uplink path) was much lower than in the early experiments employing bondwires. 

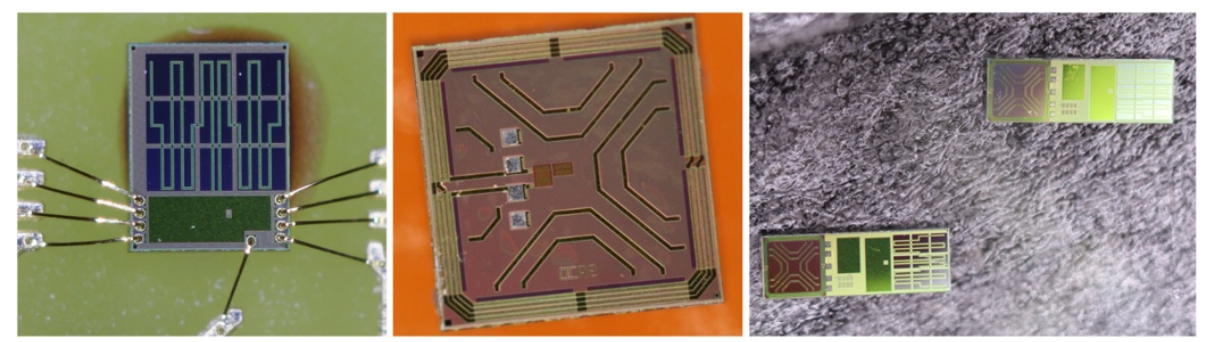

Figure 1 Microscopic images of on-chip antennas (OCAs). From left to right: external powered PPM UWB modulator front end with a meandered dipole on-chip antenna [13]; loop antenna on chip for inductive coupling [14]; prototype of a passive tag with two OCAs [15].

\section{Functionality of the system on chip}

The device under test shown in detail in this contribution is a full system on chip. A transmitter, a receiver, a power harvester, and antennas are integrated within one host platform, which is placed on one piece of silicon. The device used for short-range connectivity were manufactured with a standard $130 \mathrm{~nm}$ CMOS technology. A loop structure and an electric monopole were integrated on the chip as can be seen in the chip photograph and antenna schematic in Figure 2. The loop structure is connected to a harvester and extracts the necessary energy to drive the circuit from a UHF signal by inductive coupling. The monopole, placed inside the loop antenna, is a meandered line which transmits the UWB pulses. With respect to the overall size of the $\mathrm{SoC}(3.5 \mathrm{~mm} \times 1 \mathrm{~mm})$, the antenna part is small $\left(1 \mathrm{~mm}^{2}\right)$. The circuit on chip is programmable via the UHF link, and the signal sent on the uplink can be selected to be a continuous wave, a data sequence of pulses, or a continuous pulse stream. For characterising the device under test, the flexibility to be able to select different waveforms is helpful to detect the low-power transmitted signal. Compared to other work with OCAs and UWB transmitters [16] in the field, this SoC harvests and stores energy as a system and is operated and controlled without any wired contact [2].

\section{On-chip antennas}

In the early research of on-chip antennas, these antennas were considered as replacements of wired clock distribution [17] or as wireless interconnects on chip $[18,19]$. OCAs are very common in the millimeter-wave region [20] because this is their native environment.

In the chip presented, a meandered monopole is placed inside a loop antenna, and both are located within the chip area. The antennas are placed on the chip in a way that no electrical circuits are below the antenna. The structure is shown in top of Figure 2. To support the monopole, the remaining part of the silicon is covered with metal and connected to the chips' $V_{\mathrm{SS}}$, which acts as the ground plane. A single-ended antenna is used to avoid a differential power amplification in the front end. This reduces the overall power consumption.

The silicon substrate thickness is $220 \mu \mathrm{m}$, with the conductivity being larger than $50 \mathrm{~S} / \mathrm{m}$. In [21], where exemplary dipoles have been simulated on a CMOS layer stack, it has been shown that the lossy substrate reduces the Q-factor of the antenna and therefore supports a wideband communication scheme, even when the aperture of the antenna is very small.

All antennas are placed in a so-called inductive layer in which metal filling structures between the turns are avoided. The width of the conductor used to form the monopole is $15 \mu \mathrm{m}$ with a total unwrapped length of $4.85 \mathrm{~mm}$ which is approximately $\lambda / 10$. The distance between each turn is $90 \mu \mathrm{m}$. With increasing distance to the excitation port, the width of the meandered monopole increases from 90 to $800 \mu \mathrm{m}$ in eight steps. For the uplink and the downlink antenna, three metal layers each are connected in parallel by vias to increase the thickness of the conductor. The magnetic loop antenna with four turns and chamfered edges is square with a width of $989 \mu \mathrm{m}$. The conductor used for the loop has a width of $15 \mu \mathrm{m}$ and a spacing of $2.6 \mu \mathrm{m}$. With a method of moments (MOM) simulator (Sonnet Software, North Syracuse, NY, USA) the inductance of the loop was calculated to be $50.2 \mathrm{nH}$, and the impedance of the meandered monopole was determined as $Z_{\text {uplinkMOM }}=(11.6-6.6 j) \Omega$. The accurateness of the simulation result is dependent on the physical size of the antenna and the simulation method itself, especially for small wires such as the conductors for the OCAs [22]. Therefore, and for further investigations, the meandered monopole was simulated with a finite element method (FEM) solver (HFSS - Ansoft, Ansys, Inc., Canonsburg, PA, USA). The impedance thus obtained is $Z_{\text {uplinkFEM }}=(23.4+3.66 j) \Omega$. The real part of the numerically calculated impedance from the FEM simulation is larger because the metallic antenna parts must be modelled as solid aluminium. This is in contrast to the MOM simulation, where all thin metal layers are accurately modelled as a combination of copper and aluminium. Therefore, the impedance's real part is lower in 


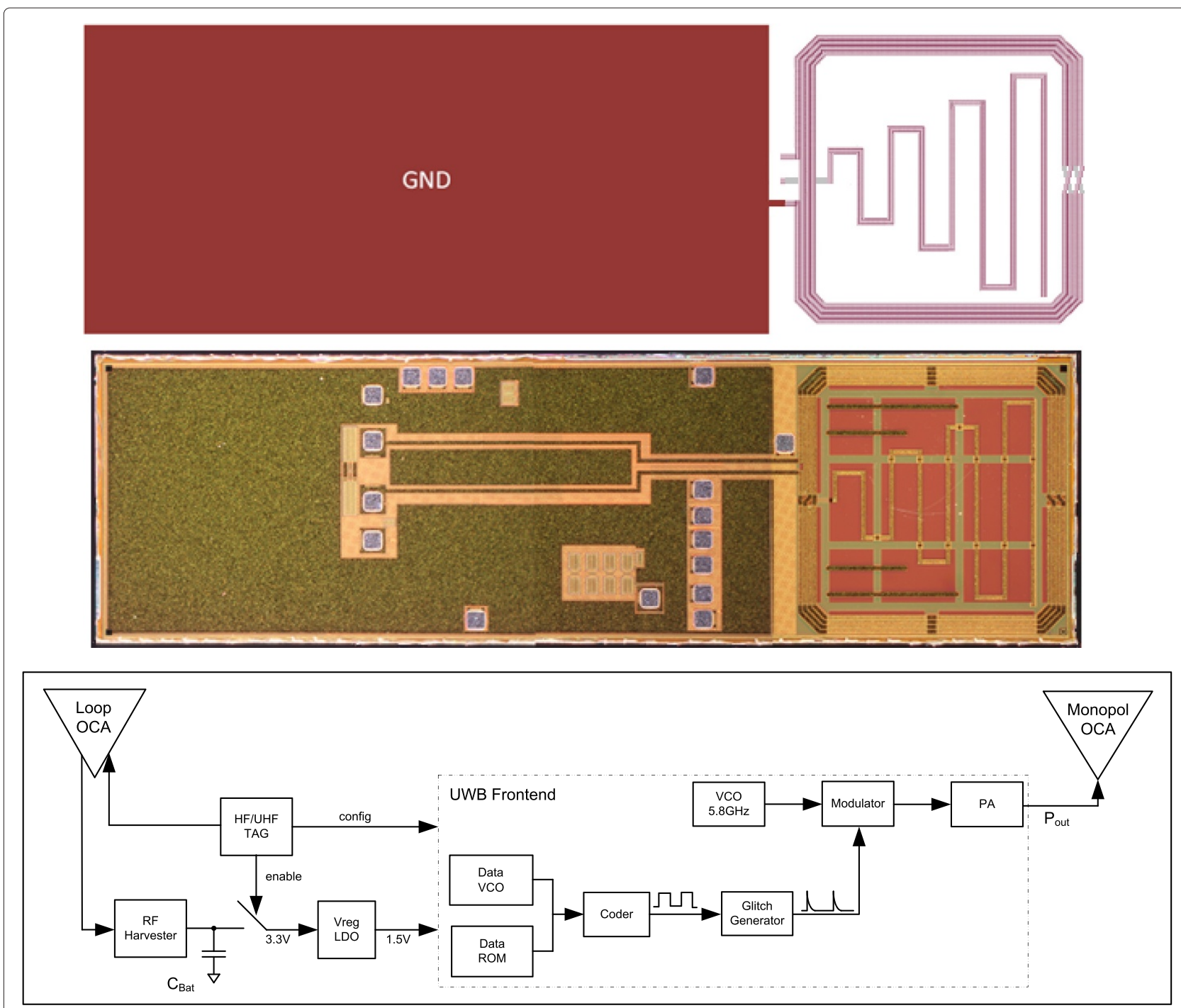

Figure 2 The silicon under test is a UHF/UWB hybrid system on chip with on-chip antennas. From the top to the bottom, a drawing of the antennas, a microscope picture of the system on chip, and a functional schematic of the integrated circuit.

the MOM simulation. In comparison to theoretical predictions for electrically small antennas, the real part is very much dominated by the losses due to the conductive silicon substrate underneath.

The far field gain of the monopole is determined to be $-41.5 \mathrm{dBi}$ with an efficiency of $-43.56 \mathrm{~dB}$ at the targeted centre frequency of $5.8 \mathrm{GHz}$. The gain flatness is $0.9 \mathrm{~dB}$ from 5 to $6.2 \mathrm{GHz}$ which is important for pulse transmission.

\section{Integrated circuit}

In the downlink from the reader to the miniature device, a standard UHF RFID EPC protocol is used. The communication frequency is $f_{c}=866 \mathrm{MHz}$. A power harvester [23] stores the energy in a buffer capacitor with a capacitance of $C_{\mathrm{Bat}}=3.9 \mathrm{nF}$. The HF rectifier of [23] is replaced by a standard UHF rectifier. For the functionality of this component, it is not important if the tag is placed in the near or far field of a reader antenna. Moreover the used electric or magnetic antenna connected to rectifier is important, so that the tag can be operated either in the near or far field of a reader. The harvester needs a minimum input of $P_{\min }=-15 \mathrm{dBm}$ for operation. The DC current consumption of the tag is $8.5 \mu \mathrm{A}$. Simulations show that a power of $P=-10 \mathrm{dBm}$, not needed by the transponder circuitry, is redirected into the charge pump, placed before the buffer capacitor. A schematic of the tag is shown in the bottom of Figure 2.

Once the capacitor is fully charged and the command to transmit the hardwired sequence is triggered by the RFID reader, pulses with a nominal width of either $w_{\text {low }}=$ $0.78 \mathrm{~ns}$ or $w_{\text {high }}=1.3 \mathrm{~ns}$ are created by the glitch 
generator and transmitted by the monopole antenna. The uplink frequency is $f_{c}=5.8 \mathrm{GHz}$. The motivation of this uplink frequency is not triggered by standardisation; furthermore, it is a trade-off between CMOS transit frequency and hence a low power consumption of the active front end. Since a carrier-based pulse radio is considered in the tag, a centre frequency within an available ISM band is feasible. A pulse-position modulation is used, where four sub frames and one guard frame form one symbol frame (4-PPM). The transmitted data sequence consists of 16 symbols, which are repeated until the capacitor reaches a minimum level of charge. In the design, the data rate was calculated to be $117 \mathrm{Mbit} / \mathrm{s}$ and the nominal symbol rate is $58 \mathrm{Msym} / \mathrm{s}$. The supply voltage of the UWB front end is $1.5 \mathrm{~V}$, controlled by a low-dropout (LDO) regulator placed before the UWB front end. The available power $P_{\text {out }}$ provided by the single-ended amplifier to the OCA monopole was simulated using Cadence software to be $-1.3 \mathrm{dBm}$. The simulated antenna impedance is connected in Cadence to the front end, and the peak output power of a continuous wave delivered by the front end is determined. In the following measurements, the pulse mode is analysed, hence the available power has to be corrected with the duty cycle of the pulse signal. Therefore, in pulse mode, with a pulse width of $w_{\text {low }}=0.78 \mathrm{~ns}$ and a duty cycle of $D=4.5 \%$, the available power fed to the OCA is $P_{\text {out }}=-28.2 \mathrm{dBm}$.

\section{Measurements with a hybrid UHF/UWB reader setup}

A photograph of the measurement setup is shown in Figure 3. A UHF RFID reader (RFID $\mathrm{ME}^{\mathrm{m}}$ USB Dongle, MTI, Inc., Hsinchu, Taiwan) acts as the signal source for the downlink path. The USB reader was programmed to be able to send control commands and select the different operating modes of the silicon under test.

Since the pulse burst transmitted by the silicon is as short as $10 \mu \mathrm{s}$, a triggering event is required. Therefore, the initial rising edge of the UHF signal is sampled by a coupler and consequently used as trigger source for the oscilloscope. Following the coupler two lowpass filters in series suppress the spurious harmonics produced by the UHF reader.

The scenario is shown in the subfigure in Figure 3: The downlink excitation coil is printed on a standard FR4 substrate and is matched to the downlink path (centre frequency $866 \mathrm{MHz}$ ). This external loop antenna replaces the internal antenna of the UHF reader. In this way, we have more degrees of freedom for characterising the device under test. The UWB silicon chip is fixed with adhesive tape in close vicinity to the excitation coil. We wish to point out, however, that the chip has not a single conductive connection to any part of the measurement equipment.
A reference antenna was positioned in a distance of $d_{\text {uplink }} \approx 5 \mathrm{~mm}$ above the silicon, capturing the silicon's uplink. This reference antenna is also a loop structure with one turn of copper wire; designed to be resonant at the uplink frequency of $5.8 \mathrm{GHz}$. The $\mathrm{SoC}$, the UHF excitation coil, and the reference antenna were located inside a brass box to suppress the local WLAN and other interferers.

As shown in schematic view of the measurement setup (Figure 3), a chain of bandpass filters and amplifiers is used before the signal is fed to a digital sampling oscilloscope.

The components of the uplink, such as the reference coil and the two parallel coupled half-wave resonators, are measured and the results presented in Figure 4. All passive components have been simulated and measured with respect to a reference of $50 \Omega$. The transmission coefficients of the bandpass filters are $G_{\mathrm{BP} 1}=-1.3 \mathrm{~dB}$ and $G_{\mathrm{BP} 2}=-1.4 \mathrm{~dB}$. The influence of the splitter and the cables is summarised in $G_{\mathrm{sc}}=-7.5 \mathrm{~dB}$. The two amplifiers have a gain of $G_{\mathrm{ZX} 60}=12.3 \mathrm{~dB}$ and $G_{\mathrm{ZVA} 183+}=$ $27.2 \mathrm{~dB}$. The amplification of the total uplink path is

$$
G_{\text {meas }}=G_{\mathrm{ZX} 60}+G_{\mathrm{ZVA} 183+}+G_{\mathrm{BP} 1}+G_{\mathrm{BP} 2}+G_{\mathrm{sc}}
$$

which results in $G_{\text {meas }}=29.3 \mathrm{~dB}$. The power at the oscilloscope or spectrum analyser can be written as

$$
P_{\text {meas }}=G_{\text {meas }}+L_{\text {uplink }}+P_{\text {out }} .
$$

$L_{\text {uplink }}$ describes the transmission between the monopole and the reader antenna, including the gain and possible mismatch of both antennas.

\section{Frequency domain measurement}

A spectrum analyser was used to characterise the uplink in the frequency domain. A resolution bandwidth of $10 \mathrm{MHz}$ and a video bandwidth of $50 \mathrm{kHz}$ were set. Since the impulse radio event of the tag is short in time and the sweep time of the analyser is too long to capture a transmission precisely, the max hold functionality was set and 32,500 sweeps were captured. The result of this measurement is shown in Figure 5. We observe that the harmonics of the $866 \mathrm{MHz}$ downlink signal are present at $5,196 \mathrm{MHz}$ and $6,062 \mathrm{MHz}$, indicating that more suppression in the downlink path of the measurement setup is necessary. The tag is set to transmit continuous pulses (constant pulse repetition rate), hence a comb line spectrum is expected. This spectrum is visible at a centre frequency of $f_{\mathrm{c}}=$ $5.64 \mathrm{GHz}$, which differs only slightly from the targeted design frequency due to CMOS process variation. The spectral line at this centre frequency is $13 \mathrm{~dB}$ above the noise floor. The $10 \mathrm{~dB}$ bandwidth of the pulsed signal is $230 \mathrm{MHz}$. The coil antenna used for receiving the pulse signal is narrowing the observed frequency band. This can be seen in Figure 4 with the plotted reflection coefficients of the reference coil. 


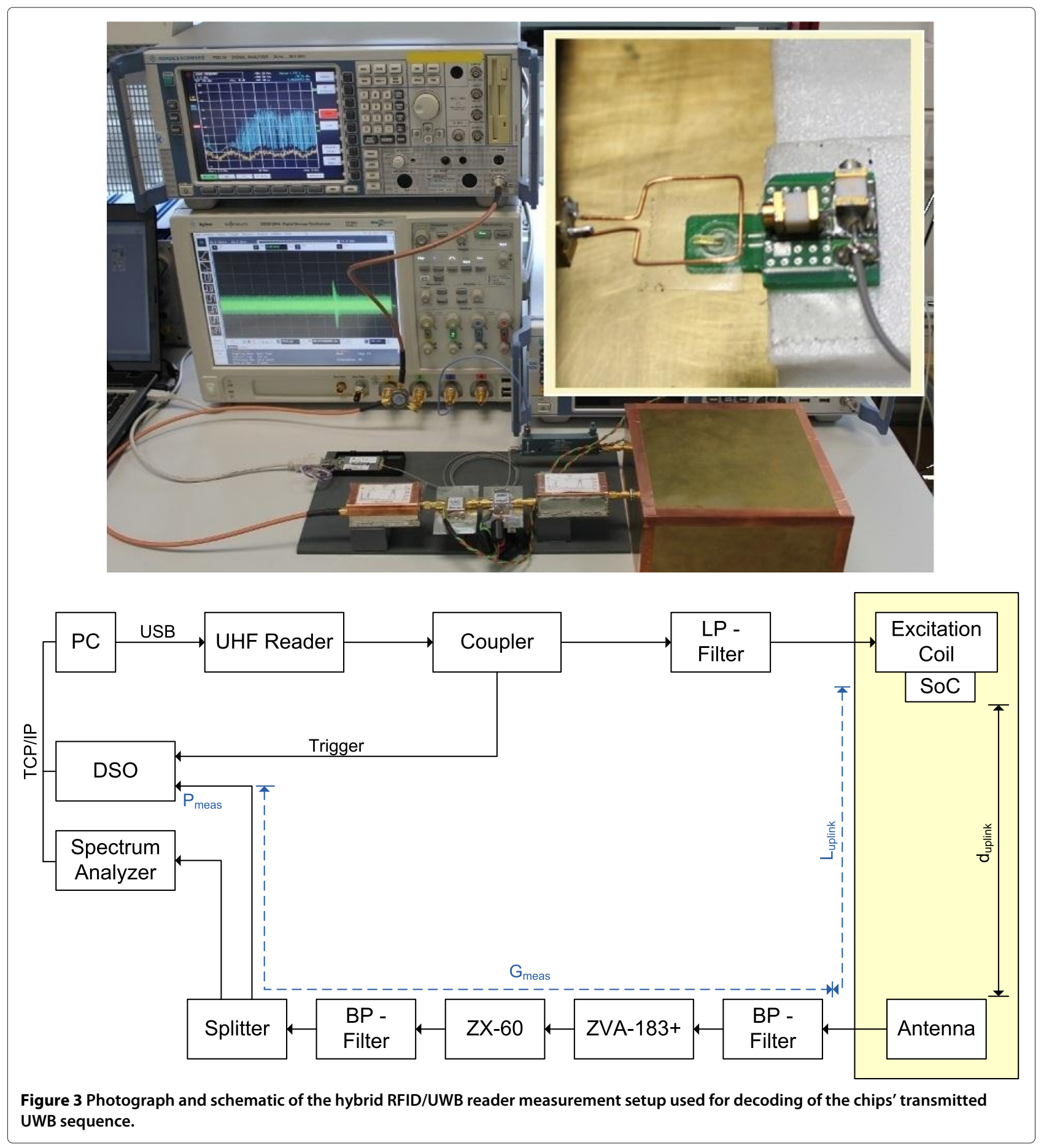

\section{Time domain measurement}

Two captures with a digital storage oscilloscope $(40 \mathrm{GSa} / \mathrm{s})$ as shown in Figure 6 were taken. In part (a) of Figure 6, a continuous stream of fixed 4-PPM symbols is presented. A 16-symbol data like sequence is shown in part (b). For both, the complete burst is shown on the left side. The right side is a window zoomed into the respective waveforms. The continuous pulse stream has an average frame length of $15.8 \mathrm{~ns}$, which yields to a data rate of $63.3 \mathrm{MSym} / \mathrm{s}$. Comparing the envelope of the burst amplitude of the continuous signal and the data sequence, one can observe that the latter is almost constant in time between 0 and $8 \mu \mathrm{s}$, whereas the amplitude of the continuous signal is initially higher and decreases with time. 


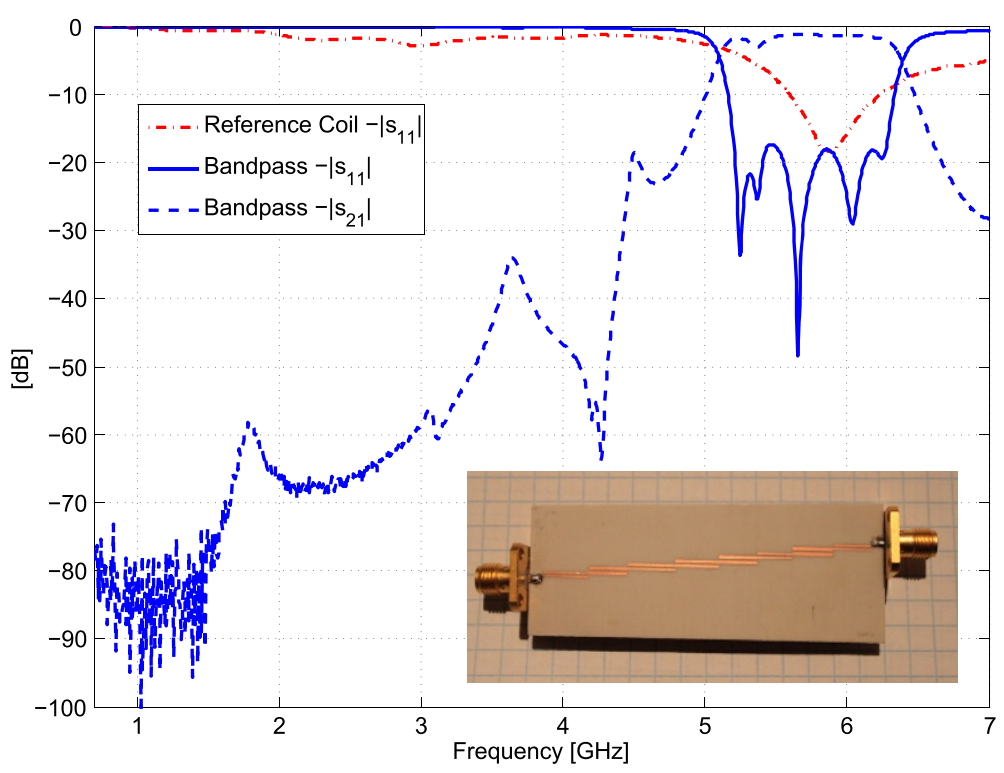

Figure 4 Logarithmic transmission and reflection coefficients of reference coil and bandpass filters. These were used in the uplink path of the measurement setup.

From transient simulations, it is known that at $\approx 8 \mu \mathrm{s}$, the initial voltage of $3.3 \mathrm{~V}$ stored in the buffer capacitor discharges below the minimal supply voltage of $1.5 \mathrm{~V}$, required by the UWB front end. The average amplitude of the continuous pulsed signal is higher than that of the waveform with the data sequence. The amplitude of the preamble, which is based on five continuous pulses, is higher than the amplitude of the following data sequence.

\section{Decoding of the UWB uplink 4-PPM signal}

In this section, the 4-PPM signal is decoded step by step in a Matlab post processing step. The oscilloscope captured the waveform of the transmitted pulses, with the pulse width set to $w_{\text {low }}$ in the data sequence mode. Not to waste processing time, from the received signal $r(t)=s(t)+n(t)$ the pulse burst is extracted. This burst is squared and filtered with a Matlab FIR equiripple bandpass filter. The

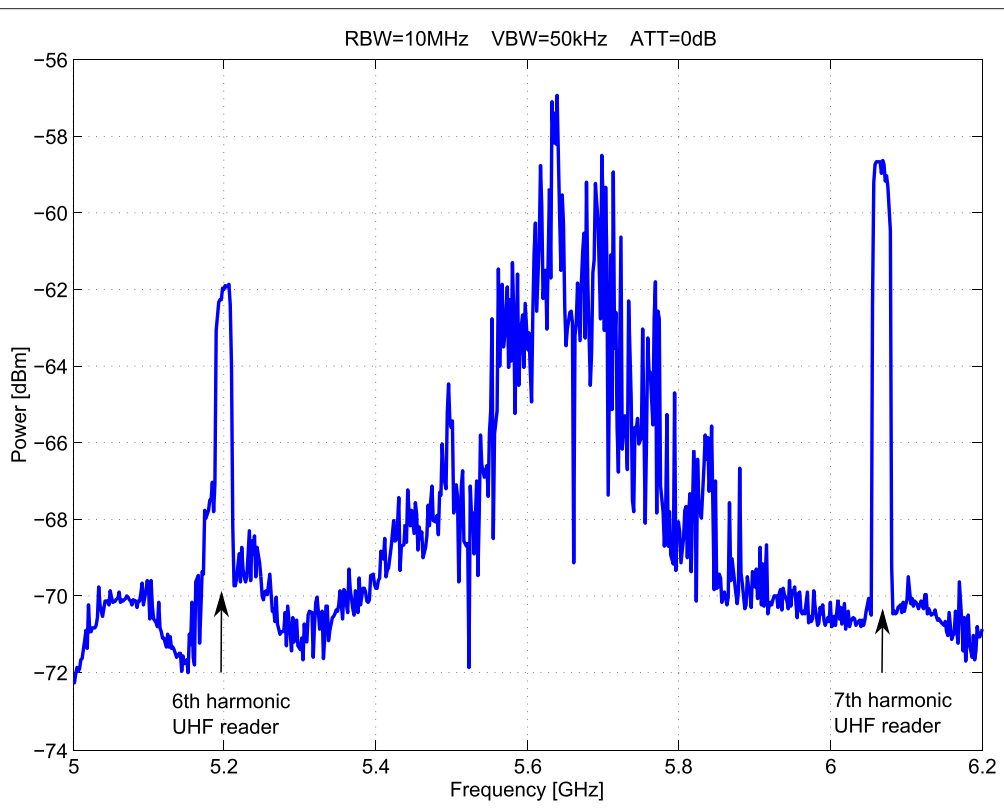

Figure 5 Measurement of wideband uplink signal with harmonics of narrowband downlink signal in frequency domain. 
a)

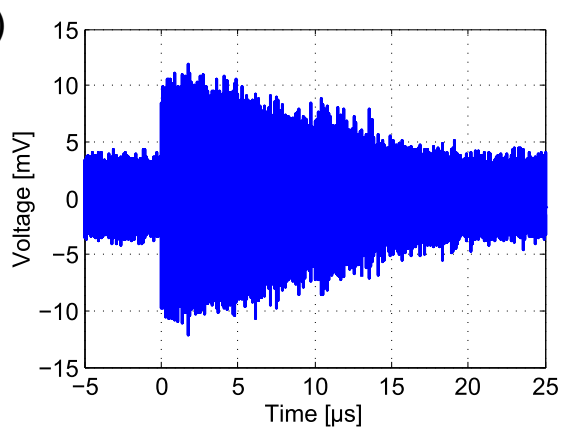

b)

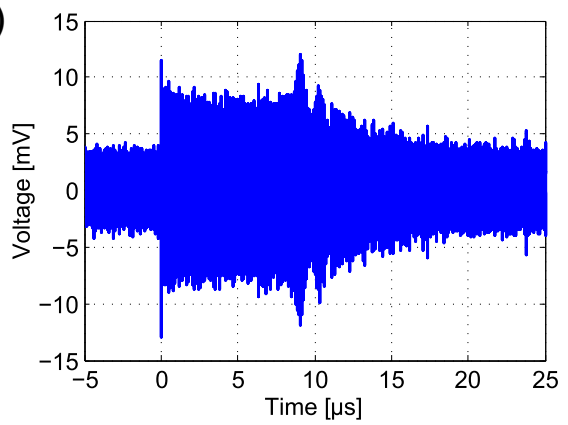

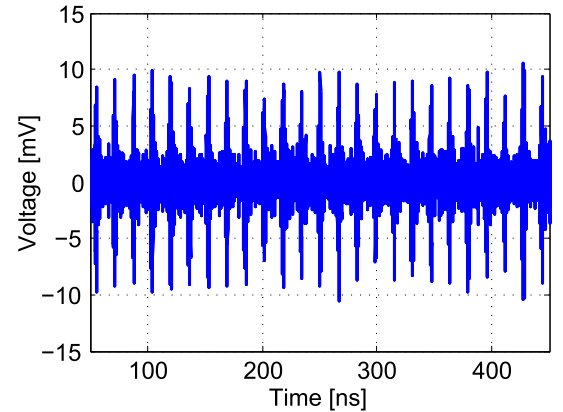

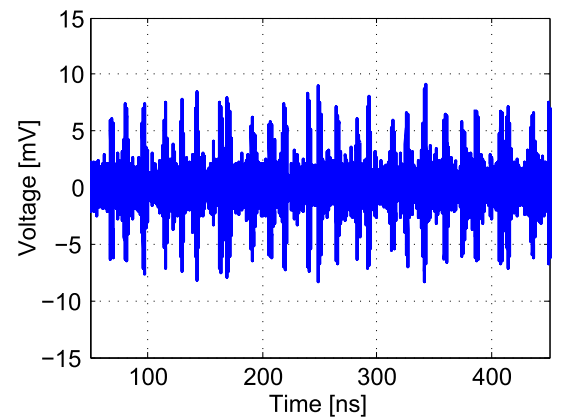

Figure 6 Captured voltage waveforms in the time domain. With amplitude of the miniature wireless chip transmitting a continuous (a) and data (b) sequence.

filter has a centre frequency of $5.6 \mathrm{GHz}$ and a $60 \mathrm{~dB}$ bandwidth of $1.6 \mathrm{GHz}$. Now, the signal is squared again and subsequently normalised to the maximum peak.

$$
\begin{aligned}
& r^{\prime}(t)=\left(r(t)^{2} * h_{\mathrm{BPF}}\right)^{2} \\
& x(t)=\frac{1}{\max \left(r^{\prime}(t)\right)} \times r^{\prime}(t)
\end{aligned}
$$

The normalised signal $x(t)$ is shown in Figure 7a. In this representation, one can observe a deep fade in the amplitude at around $10 \mu \mathrm{s}$. At this instant, the voltage supplied by the on-chip capacitor becomes too weak for the UWB front end to operate.

The decoder itself is based on determining the time between the current pulse and the following pulse, taking into account the estimated symbol corresponding to the current pulse. Since a preamble of five symbols is transmitted at the beginning of the pulse burst, an initial state for decoding is given. In general, the amount of possible pulse distances $n_{\Delta}$ in an M-ary pulse position modulation scheme is given by

$$
n_{\Delta}=2 M-1 .
$$

The pulse distances are calculated after a threshold comparator. In Figure $7 \mathrm{~b}$ the pulse distances $t_{\Delta}$ of the first 600 symbols $(\approx 10 \mu \mathrm{s})$ are shown as a histogram. It shows the occurrence of pulse distances within the captured pulse burst. The amount of pulses having a pulse distance of $15.8 \mathrm{~ns}$ is maximum across all pulse distances. It represents consecutive equal symbols in the modulation scheme and being the frame length of the 4-PPM transmission. Only $n_{\Delta}=6$ distances can be found in the burst. The reason is that one out of seven possible transitions is not present in the hardwired 4-PPM transmitted data. Clear peaks can be observed in this representation. We conclude that no significant timing drift is caused by a decreasing supply voltage to the UWB front end.

All occurring pulse distances $t_{\Delta}$ in the data sequence of the burst are plotted versus symbols together with the average $t_{\Delta}$ in Figure $7 \mathrm{c}$. In this graph, one can observe that not only the frame rate $\left(t_{\Delta}=15.8 \mathrm{~ns}\right)$ but all other pulse distances are constant until 620 symbols. The pulse distances of all following symbols drift away and a successful decoding is not possible any more.

In the following, the transmitted symbols are estimated, with estimation borders positioned between the average pulse distances. The symbol error rate is calculated for pulse burst transmissions of several consecutive measurements. The result is shown in Figure 8 as symbol error versus decoded symbols. In most cases, a transmission 


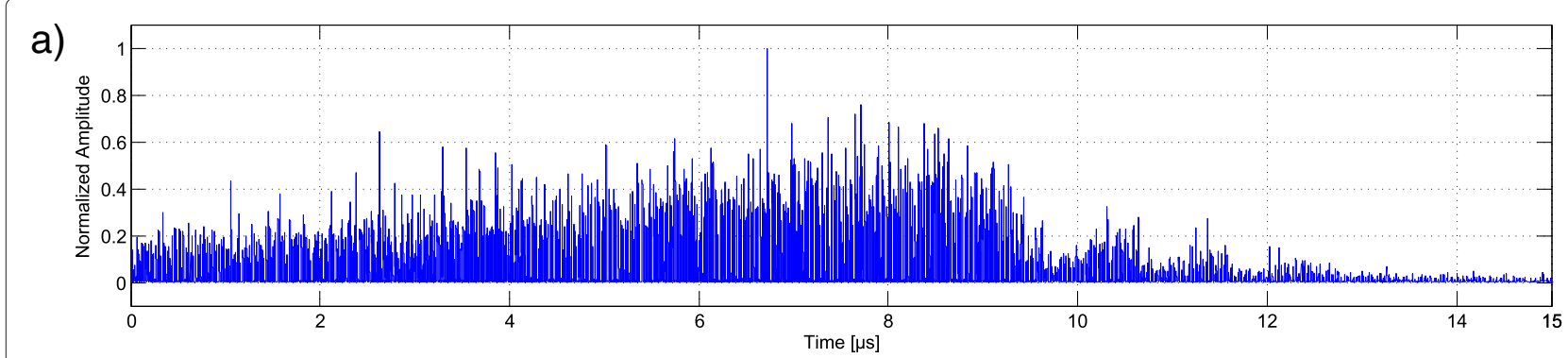

b)

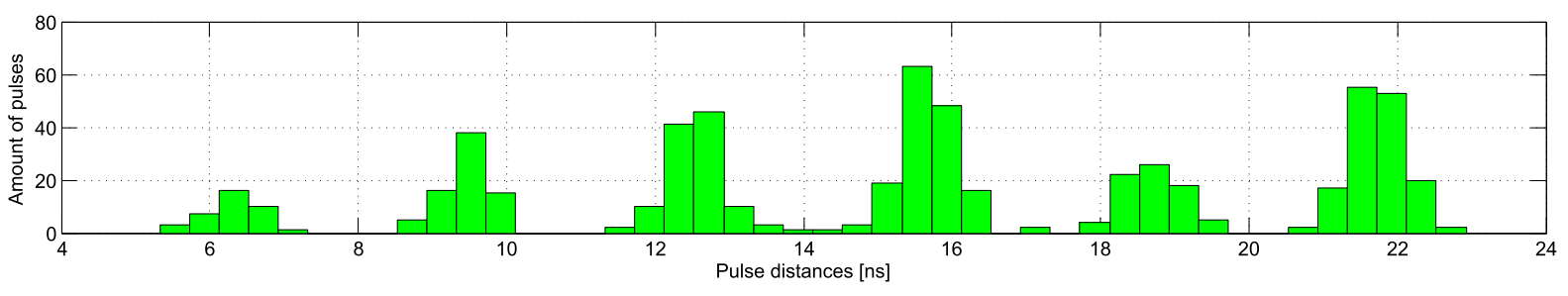

c)

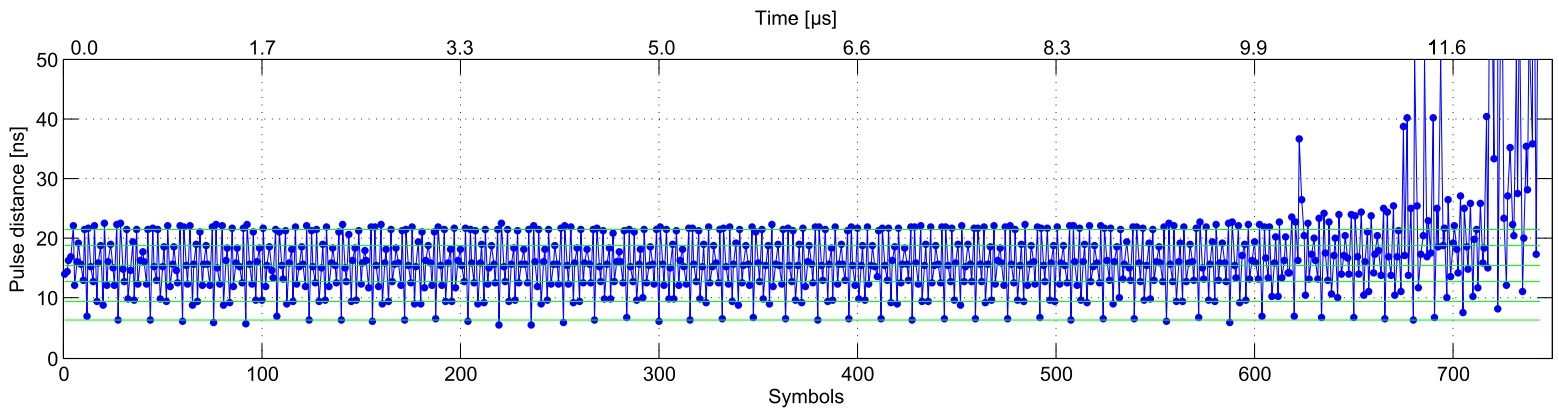

Figure 7 Amplitude of pulse burst, pulse distances of transmitted pulse burst, and pulse distances versus transmitted symbols. Filtered and normalised amplitude of the pulse burst (a), histogram of pulse distances $t_{\Delta}$ of a transmitted pulse burst (b), and the pulse distances versus transmitted symbols (c).

without errors is possible until 600 symbols (equal to 1,200 bits). The theoretical and experimental limitation to decode up to the given number of symbols is given through the finite size of the buffer capacitor of the SoC, and the power efficiency of the TX-UWB front end, being the only consumer.

\section{UWB OCA transmission loss}

The received power $P_{\text {meas }}$ can be calculated with the root mean square voltage of the signal and the duty cycle of the impulse signal [24]:

$$
\begin{aligned}
U_{\mathrm{RMS}} & =\frac{U_{P}}{\sqrt{2}} \times D \\
P_{\text {meas }} & =\frac{U_{\mathrm{RMS}}^{2}}{R}=\frac{1}{2 R} \times\left(\frac{U_{P} \times T}{\tau}\right)^{2} .
\end{aligned}
$$

With an average pulse amplitude of $U_{p}=8 \mathrm{mV}$ and a duty cycle of $D=T / \tau=2.6 \mathrm{~ns} / 15.8 \mathrm{~ns}=0.16$ it is $P_{\text {meas }}=-47.6 \mathrm{dBm}$. The transmission coefficient between the monopole and the readers' loop antenna according to this measurement is $L_{\text {uplink }}=-48.7 \mathrm{~dB}$.

\section{Comparison with related work}

The figures of merit of the presented device using a UHF/UWB asymmetric communication scheme are compared to similar devices in Table 1. It has to be mentioned that the device from [16] is powered externally, and the devices in [25] and [6] use external antennas. In this table, the required power $P_{\text {downlink }}$ necessary to establish a wideband connectivity over the distance $d_{\text {downlink }}$ is used as a basis of comparison.

\section{Conclusions}

In this contribution, the potential use of on-chip antennas for RFID tags with ultra-wideband signalling is investigated by a prototypical implementation. The $\mathrm{SoC}$ is described in detail featuring two on-chip antennas and an asymmetric wireless communication scheme.

The measurement setup is presented which comprises a commercially available UHF RFID dongle and a spectrum analyzer as well as a digital storage oscilloscope. An optimisation is carried out in the uplink path for increasing the signal-to-noise ratio of the received signal using 


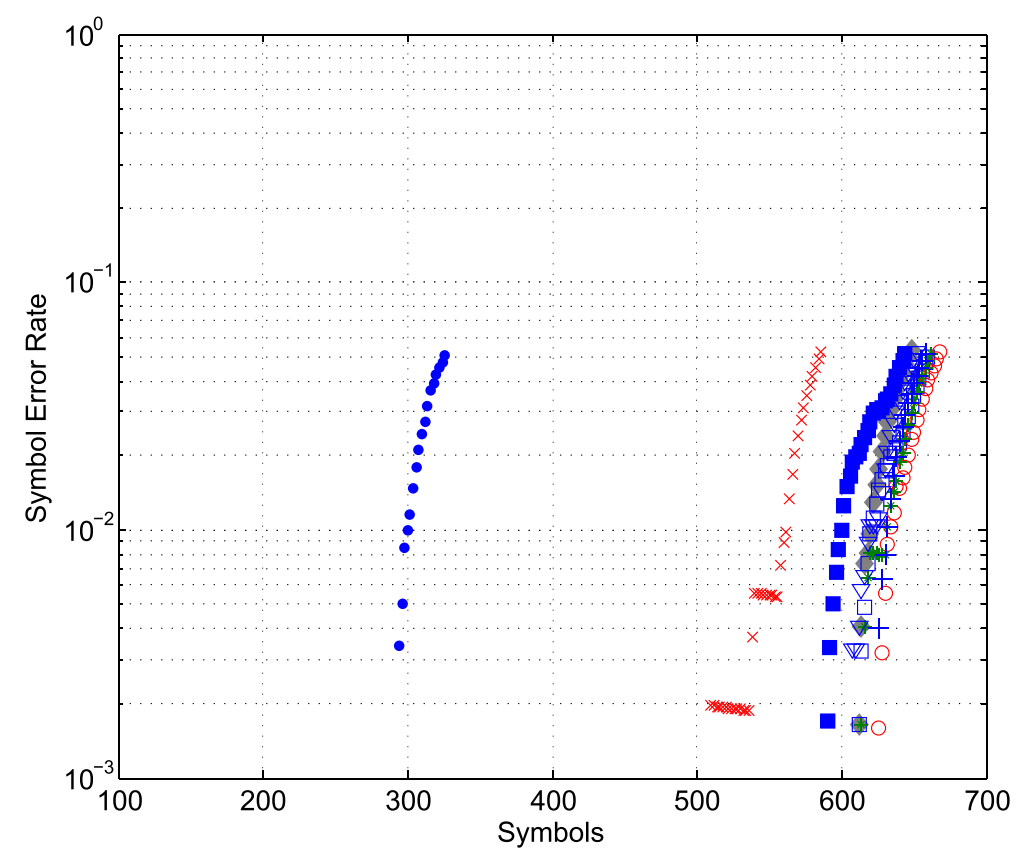

Figure 8 Symbol error rate versus transmitted symbols. For nine consecutive UWB data transmissions from SoC to reader antenna.

Table 1 Important figures of merit for RFID tag with hybrid communication scheme in comparison to similar research

\begin{tabular}{|c|c|c|c|c|c|c|}
\hline Path & Parameter & This work & Radiom [3] & Kulkarni [16] & Pelissier [25] & Vauche [6] \\
\hline & Technology & $130 \mathrm{~nm}$ & $180 \mathrm{~nm}$ & $180 \mathrm{~nm}$ & $130 \mathrm{~nm}$ & $130 \mathrm{~nm}$ \\
\hline & Die-Size & $3.5 \mathrm{~mm}^{2}$ & $4.5 \mathrm{~mm}^{2}$ & $>5.4 \mathrm{~mm}^{2}$ & $4 \mathrm{~mm}^{2}$ & $0.8 \mathrm{~mm}^{2}$ \\
\hline \multirow[t]{6}{*}{ Downlink } & OCA & Yes & Yes & - & Ext. antenna & Ext. antenna \\
\hline & $f_{\text {downlink }}$ & $866 \mathrm{MHz}$ & $5.8 \mathrm{GHz}$ & - & $900 \mathrm{MHz}$ & $900 \mathrm{MHz}$ \\
\hline & Receiver & Yes & Yes & - & Yes & UHF and UWB \\
\hline & Antenna gain & - & $-29.5 \mathrm{dBi}([2])$ & - & - & $0 \mathrm{dBi}$ \\
\hline & $P_{\text {downlink }}$ & $13 \mathrm{dBm}$ & $36 \mathrm{dBm}$ & - & $14 \mathrm{dBm}$ & $36 \mathrm{dBm}$ \\
\hline & $d_{\text {downlink }}$ & $0 \mathrm{~mm}$ & $75 \mathrm{~mm}$ & - & $100 \mathrm{~mm}$ & $10 \mathrm{~m}$ \\
\hline Harvester & Capacitor & $3.9 \mathrm{nF}$ & $6 \mathrm{nF}$ & - & - & $56 \mathrm{pF}$ \\
\hline \multirow[t]{9}{*}{ Uplink } & OCA & Yes & Yes & Yes & Ext. antenna & Ext. antenna \\
\hline & $f_{\text {uplink }}$ & $5.64 \mathrm{GHz}$ & $5.8 \mathrm{GHz}$ & $8 \mathrm{GHz}$ & $7.9 \mathrm{GHz}$ & $7.25 \mathrm{GHz}$ (sim) \\
\hline & Antenna gain & $-41.5 \mathrm{dBi}$ & - & $-27.4 \mathrm{dBi}$ & - & $-(\operatorname{sim})$ \\
\hline & IR-scheme & 4 PPM & OOK / BPSK & BPM & OOK & Localisation (sim) \\
\hline & Data rate & 126.6 MBit/s & $1 \mathrm{MBit} / \mathrm{s}$ & $750 \mathrm{MBit} / \mathrm{s}$ & $112.5 \mathrm{Mbit} / \mathrm{s}$ & $200 \mathrm{Mbit} / \mathrm{s}$ (sim) \\
\hline & Pulse width & $2.6 \mathrm{~ns}$ & $1 \mathrm{~ns}$ & $0.5 \mathrm{~ns}$ & $2.9 \mathrm{~ns}$ & 0.45 ns (sim) \\
\hline & $P_{\text {uplink }}$ & $-28.2 \mathrm{dBm}$ & - & $-42 \mathrm{dBm}$ & $-16.6 \mathrm{dBm}$ & - \\
\hline & $d_{\text {uplink }}$ & $5 \mathrm{~mm}$ & $75 \mathrm{~mm}$ & - & 100 mm & - \\
\hline & Bits per burst & 1,248 & - & - & - & $1(\operatorname{sim})$ \\
\hline
\end{tabular}


bandpass filters and amplifiers. Further, interferers in the downlink path (e.g. local WLAN and harmonics of the powering UHF signal) had to be suppressed.

Bursts of nanosecond pulses from the UHF/UWB hybrid silicon RFID tag were captured and analysed with the optimised measurement setup. The time domain and frequency domain measurements were subsequently analysed by post processing. Due to the short transmission time, the low duty cycle and the low transmit power of the signal, we found that it is easier to detect the radiated signal in the time domain. The ability to switch the SoC between different operating modes for data transmission enhances the initial signal detection.

The transmitted ultra-wideband data sequence from the tiny SoC is decoded correctly up to sequence lengths of 1,248 bits. The embedded monopole OCA broaden the pulse width from 0.7 to $2.6 \mathrm{~ns}$. A data transmission with simulated pulse width of $1.3 \mathrm{~ns}$ is therefore not successful.

The transmission loss from a CMOS OCA to a reader antenna is estimated to be $L_{\text {uplink }}=-48.7 \mathrm{~dB}$ from transient simulations and measurements.

In comparison to related work in the field [3], the downlink distance is reduced which is reflected in a much lower power requirement for inductive coupling by $20 \mathrm{~dB}$. We conclude that Impulse Radio is a promising low-power communication scheme for a fast, secure, and powerefficient data transfer for future RFID enhancements.

\section{Competing interests}

The authors declare that they have no competing interests.

\section{Acknowledgements}

This work was performed as part of the project 'ConSens' (Contactless Sensing) within the funding programme 'Forschung, Innovation, Technologie - Informationstechnologie' (FIT-IT) of the 'Bundesministerium für Verkehr, Innovation und Technologie' (BMVIT). The authors wish to thank the members of the COST action IC 1004, "Cooperative Radio Communications for Green Smart Environments," for countless fruitful discussions.

\section{Author details}

${ }^{1}$ Institute of Telecommunications, Vienna University of Technology, Gusshausstrasse 25/E389, Vienna 1040, Austria. ${ }^{2}$ Infineon Technologies Austria AG, Contactless and RF Exploration, Babenberger Strasse 10, Graz 8020, Austria.

\section{Received: 21 December 2012 Accepted: 29 July 2013}

Published: 16 August 2013

\section{References}

1. LR Zheng, MB Nejad, Z Zou, X DS Zou, Z Zhang, H Tenhunen, Future RFID and wireless sensors for ubiquitous intelligence, in Proc NORCHIP (Tallinn, 16-17 Nov. 2008), pp. 142-149

2. M Baghaei-Nejad, S Radiom, G Vandenbosch, LR Zheng, G Gielen, Fully integrated $1.2 \mathrm{pJ} / \mathrm{p}$ UWB transmitter with on-chip antenna for wireless identification, in IEEE International Conference on Ultra-Wideband (Nanjing, 20-23 Sept. 2010), pp. 1-4

3. S Radiom, M Baghaei-Nejad, K Aghdam, G Vandenbosch, L Zheng, $G$ Gielen, Far-field on-chip antennas monolithically integrated in a wireless-powered 5.8-GHz downlink/UWB uplink RFID tag in $0.18 \mu \mathrm{m}$ standard CMOS. IEEE J. Solid-State Circ. 45(9), 1746-1758 (2010)
4. BH Calhoun, J Lach, J Stankovic, DD Wentzloff, K Whitehouse, AT Barth, JK Brown, Q Li, S Oh, NE Roberts, Y Zhang, Body sensor networks: a holistic approach from silicon to users. In Proceedings of the IEEE. 100, 91-106 (2012)

5. D Wentzloff, A Chandrakasan, Gaussian pulse generators for subbanded ultra-wideband transmitters. IEEE Trans. Microwave Theory and Tech. 54(4), 1647-1655 (2006)

6. R Vauche, E Bergeret, J Gaubert, S Bourdel, O Fourquin, N Dehaese, A remotely UHF powered UWB transmitter for high precision localization of RFID tag, in IEEE International Conference on Ultra-Wideband, (Bologna, 14-16 Sept 2011), pp. 494-498

7. P Meissner, D Arnitz, T Gigl, K Witrisal, Analysis of an indoor UWB channel for multipath-aided localization, in IEEE International Conference on Ultra-Wideband, (Bologna, 14-16 Sept. 2011), pp. 565-569

8. Y Zheng, MA Arasu, KW Wong, YJ The, AHS Poh, DD Tran, WG Yeoh, DL

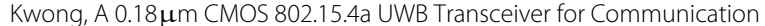
and Localization, in Solid-State Circuits Conference, (San Francisco, 3-7 Feb 2008), pp. 118-120

9. PK Gentner, GS Hilton, MA Beach, CF Mecklenbräuker, Characterisation of ultra-wideband antenna arrays with spacings following a geometric progression. IET Commun. 6(10), 1179-1186 (2012)

10. NM Gvozdenovic, PK Gentner, CF Mecklenbräuker, Antenna array for the reader of an ultra-wideband identification tag with on-chip antenna, in Loughborough Antennas \& Propagation Conference, (Loughborough, 14-15 Nov. 2011), pp. 1-4

11. M Mahfouz, G To, M Kuhn, No strings attached. IEEE Microwave Mag.. 12(7), S34-S48 (2011)

12. N Shinohara, Power without wires. IEEE Microwave Mag., 12(7), S64-S73 (2011)

13. PK Gentner, M Wiessflecker, H Arthaber, AL Scholtz, CF Mecklenbräuker, Measured wideband near-field characteristics of an UWB RFID tag with on-chip antenna, in International Conference on Ultra-Wideband, (Bologna, 14-16 Sept. 2011), pp. 479-483

14. PK Gentner, G Hofer, AL Scholtz, CF Mecklenbräuker, Accurate measurement of power transfer to an RFID tag with on-chip antenna, in Progress In Electromagnetics Research Symp, (Moscow, 18-21 Aug. 2012), pp. 227-230

15. PK Gentner, P Amreich, H Reinisch, G Hofer, A passive ultra wideband tag for radio frequency identification or wireless sensor networks, in International Conference on Ultra-Wideband, (Syracuse, 17-20 Sept. 2012), pp. 2-5

16. V Kulkarni, M Muqsith, A 750 Mb/s, 12 pJ/b, 6-to-10 GHz CMOS IR-UWB transmitter with embedded on-chip antenna. IEEE J. Solid-State Circ., 44(2), 394-403 (2009)

17. K Kim, $\mathrm{H}$ Yoon, $\mathrm{KK} \mathrm{O}$, On-chip wireless interconnection with integrated antennas, in Electron Devices Meeting, (San Francisco, 10-13 Dec. 2000), pp. $485-488$

18. Y Wang, D Makadia, M Margala, On-chip integrated antennas - the first challenge for reliable on-chip wireless interconnects, in Canadian Conference on Electrical and Computer Engineering, (Ottawa, 7-10 May 2006), pp. 2322-2325

19. T Kikkawa, PK Saha, N Sasaki, K Kimoto, Gaussian monocycle pulse transmitter using $0.18 \mu \mathrm{m}$ CMOS technology with on-chip integrated antennas for inter-chip UWB communication. IEEE J. Solid-State Circ., 43(5), 1303-1312 (2008)

20. T Yao, L Tchoketch-Kebir, O Yuryevich, Gordon M, SP Voinigescu, $65 \mathrm{GHz}$ Doppler Sensor with On-Chip Antenna in $0.18 \mu \mathrm{m}$ SiGe BiCMOS, in MTT-S International Microwave Symposium Digest (2006), (San Francisco, 11-16 June 2006), pp. 1493-1496

21. PK Gentner, A Adalan, AL Scholtz, CF Mecklenbräuker, Impact analysis of silicon and bondwires on an on-chip antenna, in European Conference on Antennas and Propagation, (Prague, 26-30 March 2012), pp. 3168-3172

22. MD Estarki, Y Xing, XU H, RG Vaughan, The effect of gap size on dipole impedance using the induced EMF method, in URSI International Symposium on Electromagnetic Theory (EMTS), Berlin, 16-19 Aug., (IEEE, Piscataway, 2010), pp. 373-376

23. H Reinisch, S Gruber, M Wiessflecker, H Unterassinger, G Hofer, W Pribyl, G Holweg, An electro-magnetic energy harvester with 190nW idle mode power consumption for wireless sensor nodes, in European Solid-State Circ. Conference, (Seville, 14-16 Sept. 2010), pp. 234-237 
24. I Oppermann, M Hämäläinen, J linatti (eds.), UWB: Theory and Applications (Wiley, West Sussex, 2004)

25. M Pelissier, J Jantunen, B Gomez, J Arponen, G Masson, S Dia, J Varteva, M Gary, A 112 Mb/s full duplex remotely-powered impulse-UWB RFID transceiver for wireless NV-memory applications. IEEE J. Solid-State Circuits. 46(4), 916-927 (2011)

doi:10.1186/1687-3963-2013-12

Cite this article as: Gentner et al:: A UHF/UWB hybrid silicon RFID tag with on-chip antennas. EURASIP Journal on Embedded Systems 2013 2013:12.

\section{Submit your manuscript to a SpringerOpen ${ }^{\mathcal{O}}$ journal and benefit from:}

- Convenient online submission

- Rigorous peer review

- Immediate publication on acceptance

- Open access: articles freely available online

- High visibility within the field

- Retaining the copyright to your article

Submit your next manuscript at $\gg$ springeropen.com 